\title{
Multimodal prehabilitation before major abdominal surgery: A retrospective study
}

\author{
Ning Qi Pang ${ }_{F R C S}$, Stephanie Shengjie $\underline{\mathrm{He}}^{2}{ }_{M B B S}$, Joel Qi Xuan $\underline{\mathrm{FoO}}^{2}{ }_{M B B S}$, Natalie Hui Ying $\underline{\mathrm{Koh}}^{2}$, Tin Wei Yuen ${ }^{2}$, \\ Ming Na Liew ${ }^{3}$, John Peter Ramya ${ }^{3}$, Yijun Loy ${ }^{4}$, Glenn Kunnath $\underline{\text { Bonney }}^{1}$ FRCS, Wai Kit $\underline{\text { Cheong }}{ }^{5}$ FRCS, \\ Shridhar Ganpathi Iyer ${ }^{1} F R C S$, Ker-Kan Tan ${ }^{5}$ PhD, Wan Chin Lim ${ }^{6}$, , Alfred Wei Chieh $\underline{\text { Kow }}{ }^{1}{ }_{F R C S}$
}

\begin{abstract}
Introduction: Prehabilitation may benefit older patients undergoing major surgeries. Currently, its efficacy has not been conclusively proven. This is a retrospective review of a multimodal prehabilitation programme.

Methods: Patients aged 65 years and above undergoing major abdominal surgery between May 2015 and December 2019 in the National University Hospital were included in our institutional programme that incorporated aspects of multimodal prehabilitation and Enhanced Recovery After Surgery concepts as 1 holistic perioperative pathway to deal with issues specific to older patients. Physical therapy, nutritional advice and psychosocial support were provided as part of prehabilitation.

Results: There were 335 patients in the prehabilitation cohort and 256 patients whose records were reviewed as control. No difference in postoperative length of stay $(P=0.150)$ or major complications $(P=0.690)$ were noted. Patients in the prehabilitation group were observed to ambulate a longer distance and participate more actively with their physiotherapists from postoperative day 1 until 4 . In the subgroup of patients with cancer, more patients underwent neoadjuvant therapy in the prehabilitation group compared to the control group $(21.7 \%$ versus $12.6 \%, P=0.009)$. Prehabilitation patients were more likely to proceed to adjuvant chemotherapy (prehabilitation $87.2 \%$ vs control $65.6 \%, P<0.001$ ) if it had been recommended.

Conclusion: The current study found no differences in traditional surgical outcome measures with and without prehabilitation. An increase in patient mobility in the immediate postoperative period was noted with prehabilitation, as well as an association between prehabilitation and increased adherence to postoperative adjuvant therapy. Larger prospective studies will be needed to validate the findings of this retrospective review.
\end{abstract}

\section{Ann Acad Med Singap 2021;50:892-902}

Keywords: Frailty, general surgery, geriatric surgery, perioperative care, prehabilitation

\section{INTRODUCTION}

Ageing is one of the biggest public health concerns of the 21 st century, presenting a challenge to the practice of medicine globally. As the Singapore population ages, research is needed to refine our knowledge in the care of older persons and the frail, so that better methods of care may be adopted for older patients with complex medical issues.
Surgery remains the standard treatment for many solid organ tumours, but older patients who are frail may be precluded from major surgery, depriving them of a chance for cure. ${ }^{1,2}$ Physical fitness, nutritional status and psychological readiness have been shown to be modifiable factors of surgical outcomes. ${ }^{3}$ In surgery, the concept of prehabilitation has emerged as a potential strategy that may help improve fitness for surgery,

\footnotetext{
${ }^{1}$ Division of Hepatobiliary \& Pancreatic Surgery, Department of Surgery, National University Hospital, Singapore

${ }^{2}$ Yong Loo Lin School of Medicine, National University of Singapore, Singapore

${ }^{3}$ Department of Nursing, National University Hospital, Singapore

${ }^{4}$ Department of Rehabilitation Medicine, National University Hospital, Singapore

${ }^{5}$ Division of Colorectal Surgery, Department of Surgery, National University Hospital, Singapore

${ }^{6}$ Department of Surgery, National University Health System, Singapore

Correspondence: Assoc Prof Alfred Wei-Chieh Kow, Division of Hepatobiliary \& Pancreatic Surgery, Department of Surgery, National University Hospital, NUHS Tower Block Level 8, 1E Kent Ridge Road, Singapore 119228.

Email:alfred_kow@nuhs.edu.sg
} 


\section{CLINICAL IMPACT}

\section{What is New}

- Prehabilitation may benefit older patients undergoing major surgeries, but its efficacy has not been conclusively proven. A retrospective review of 591 older patients in Singapore was undertaken to investigate the effect of multimodal prehabilitation on outcomes after surgery.

- To the best of our knowledge, this is the first study to report a greater adherence to adjuvant treatment in a prehabilitation cohort.

\section{Clinical Implications}

- An increase in patient mobility in the immediate postoperative period was noted with prehabilitation, as well as an association between prehabilitation and increased adherence to postoperative adjuvant therapy.

- Larger prospective studies will be needed to validate the findings of this study.

allowing patients who were otherwise too frail initially to benefit from a shot at curative treatment by improving preoperative physiological and psychological reserve. . $^{4-2}$

Although there is increasing interest in prehabilitation for frail older patients prior to major surgeries, its efficacy in improving objective clinical outcomes is still being questioned. ${ }^{13,14}$ There is a lack of robust evidence to prove the efficacy of prehabilitation. We aimed to investigate the effect of multimodal prehabilitation on postoperative outcomes in a cohort of patients who had undergone major abdominal surgery, by comparing it to a control group.

\section{METHODS}

A retrospective review to evaluate the efficacy of the Management and Innovation for Longevity in Elderly Surgical patients (MILES) programme between May 2015 and December 2019 in the National University Hospital, Singapore was conducted. The MILES programme was instituted as a preoperative prehabilitation pathway for all patients aged 65 years and above who were scheduled for elective major abdominal surgery (in the subspecialties of colorectal surgery and hepatobiliary and pancreatic surgery). All eligible patients were included in the programme. Major surgery was defined as a major resection procedure. For colorectal patients, this meant any form of colectomy (e.g. right hemicolectomy, left hemicolectomy, anterior resection and ultra-low anterior resection), reversal of ileostomy or reversal of Hartmann's procedure. For hepatobiliary and pancreatic patients, this meant any form of hepatectomy (except for simple wedge resections of segments III, IVb, V or VI) or pancreatic resections (pancreaticoduodenectomy or distal pancreatectomy with or without splenectomy).

The study was approved by the National Healthcare Group Domain Specific Review Board. Patient demographics, comorbidities, perioperative details and outcomes were extracted from the electronic medical record system and patient case notes. Perioperative data collected included preoperative haemoglobin and albumin levels, final surgical incision (fully minimally invasive as opposed to laparoscopic-assisted and open operations), postoperative analgesic requirements, participation in physiotherapy (including distance ambulated and physiotherapy activity completed on each postoperative day), complications and 30-day readmission rates. The Comprehensive Complication Index was calculated. ${ }^{15}$ Pulmonary complication was defined as either clinically diagnosed pneumonia requiring initiation of antibiotic therapy or significant atelectasis requiring supplementary oxygen therapy. In our institution, the ability to participate in physiotherapy is recorded as a range of graded exercises of increasing intensity, namely: resting in bed (RIB); sitting on edge of bed; sitting out of bed; sitting to standing (STS); moving on the spot (MOTS); ambulating; and climbing steps (CS). All patients who have undergone major surgery defined as above received physiotherapy sessions, and physiotherapy notes were available for review.

Postoperative outcomes beyond inpatient discharge were also collected. Adjuvant (chemotherapy) required was defined as the proportion of patients who were recommended chemotherapy based on Multidisciplinary Tumor Board (MTB) consensus after discussion of patient and disease factors. Initiation of adjuvant chemotherapy refers to the proportion of patients who went on to initiate adjuvant chemotherapy (regardless of date of initiation) should they be recommended by the MTB to consider adjuvant chemotherapy. Therefore, patients who declined adjuvant chemotherapy were labelled as patients who did not initiate adjuvant chemotherapy. Adherence to chemotherapy initiation date referred to the proportion of patients who managed to start their planned chemotherapy regime on time should they be recommended by the MTB to consider adjuvant chemotherapy. Completed chemotherapy 
referred to the proportion of patients who completed the entire planned course of chemotherapy as was determined prior to the initiation of adjuvant chemotherapy.

The MILES programme was set up in 2017 with the aim of improving postoperative outcomes in older patients undergoing major abdominal surgery. It incorporates aspects of multimodal prehabilitation and Enhanced Recovery After Surgery (ERAS) concepts as 1 holistic perioperative pathway to deal with issues specific to older patients. As part of the in-clinic counselling prior to surgery, patients aged 65 and above were invited to enrol in the MILES programme. All participants were first assessed by a specialist nurse with training in perioperative geriatric care. A physician specialising in geriatric preoperative assessment reviewed patients with 3 or more comorbidities and frail patients with 2 or more comorbidities. Preoperative optimisation plans, for blood pressure or glucose control for example, were laid out during these consultations. An assessment of frailty was also made, with documentation of the Edmonton Frail Scale, ${ }^{16,17}$ 3-Minute Nutrition Screening (3-MinNS) $s c o r e,{ }^{18}$ and hand grip strength. Referrals to the physiotherapist and/or nutritionist were made as appropriate, depending on the assessment by the specialist nurse or physician. All patients who scored 6 and above on the Edmonton Frail Scale were referred to the physiotherapist. Patients who scored less than 6 on the Edmonton Frail Scale but deemed to be weak in physical function as assessed by the specialist nurse/ physician, or had self-reported functional decline (e.g. fall or near-fall within 1 month) were also referred. If referred, the physiotherapist assessed the patient and recommended tailored exercises for physical prehabilitation. Aerobic, strengthening and balancing exercises were taught and practised over a 45 -minute session. Patients were encouraged to return to the hospital for follow-up exercise sessions. These exercises were also taught to patients and caregivers so that they may be carried out at home, as some patients declined to return to the hospital for further exercise sessions.

Patients who scored 2 and above on the 3-MinNS score were referred to the nutritionist, who then counselled and advised preoperative diet modification and supplementation tailored to individual patient needs. The nutritionist would first score a patient with the 7-point Subjective Global Assessment to evaluate degree of malnourishment. ${ }^{19,20}$ This tool served also as an evaluation metric for the patient's nutritional status throughout the perioperative period. A detailed dietary history was taken, and the baseline caloric and protein intake estimated. Caloric requirement was determined by Schofield equation with a stress factor of at least 1.2. Protein requirement was calculated as $1.3 \mathrm{~g} / \mathrm{kg}$ body weight, keeping in mind certain comorbidities that may require further adjustments such as chronic kidney disease. The amount of nutritional supplementation required to achieve caloric and protein targets was then calculated. This was prescribed in the form of polymeric milk formulas and myotein powder supplements. Specific milk formula prescribed depended on background comorbidities of patients.

In addition to physical and nutritional prehabilitation, detailed counselling on what to expect during the perioperative period was carried out by a specialist nurse, with the aim of allaying fear and anxiety in older patients scheduled to undergo a major operation. This included a recap of clinic discussions on the indications and potential benefits of the surgery, the likelihood of recovering in an intensive care or high dependency unit, and the expected overall length of stay. Critically, the management of postoperative pain was discussed, including the use of patient-controlled analgesia and how to use them. The benefits of active participation in postoperative physiotherapy were emphasised to patients to give them a sense of control and ownership of their condition and postoperative recovery. The technique of good incentive spirometry was taught, and an incentive spirometer was dispensed to patients for practice at home. A prehabilitation workflow is represented in Fig. 1.

Using historical data prior to the inception of the MILES programme, all patients aged 65 years and above who underwent major abdominal operation from May 2015 to December 2016 were included as a control group. Patients in the control group followed ERAS pathways (without prehabilitation), as the evidence for ERAS has been well established by then. Perioperative outcomes were compared between the MILES group and control group. As all patients in the MILES programme underwent hepatobiliary, pancreatic, or colonic resections, a similar group of patients in the control group was used for comparison to minimise selection bias. There were no significant changes in the physiotherapy protocol, ERAS protocols, or pain management strategies throughout the study period.

\section{Statistical analysis}

Statistical analyses were performed using SPSS Statistics software version 26 (IBM Corp, Armonk, US). Patient demographics were reported as frequencies. We defined statistical significance as $P<0.05$. Categorical variables were compared using the chi-square test or Fisher's Exact test. Continuous variables were compared using 


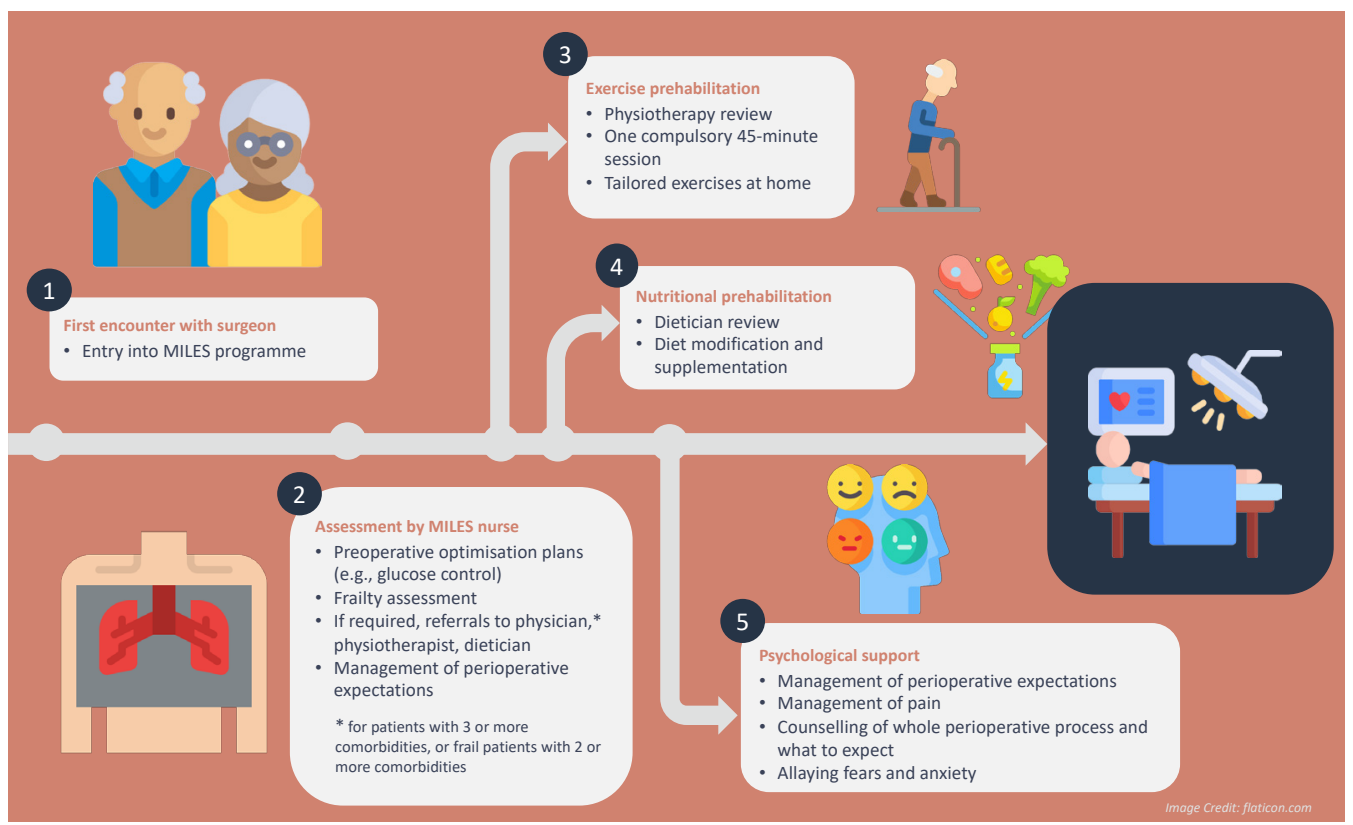

Fig. 1. Workflow of prehabilitation programme.

MILES: Management and Innovation for Longevity in Elderly Surgical patients; POD: postoperative day

paired or unpaired t-test or Mann-Whitney U test where appropriate. Disease-free survival and overall survival analyses could not be carried out as patients whose surgeries were performed until the reporting of the current study were included.

\section{RESULTS}

There were 335 patients in the MILES cohort and 256 patients in the historical control group (Table 1). There were no statistically significant differences in terms of age, sex, American Society of Anesthesiologists Physical Status Classification and most of the comorbidities, except for hyperlipidaemia $(P=0.039)$. The proportion of patients who were independent in their activities of daily living prior to surgery was also similar (MILES group 98.8\% versus control group $97.3 \%, P=0.223)$. There were more hepatobiliary and pancreatic patients than colorectal patients in the MILES group compared to control $(P=0.012)$. The number of days from surgery decision to the actual operation date was longer in the MILES group (22.5 days, standard deviation [SD] 15.7) than in the control group (17.3 days, SD 14.6) $(P=0.001)$. The median Edmonton Frail Scale score for the MILES group was 3 (interquartile range [IQR] 0-14), with 215 (64.2\%) requiring physiotherapy referral. The median 3-MinNS score was 2 (IQR 0-8) with 234 (69.9\%) patients in the MILES group referred to the nutritionist preoperatively.

The perioperative details of the 2 groups were compared in Table 2. The number of patients with a surgical incision that was minimally invasive was similar between the 2 groups (MILES group 44.6\% vs control group $46.1 \%, P=0.735)$. There were no differences in pain scores from postoperative day 1 to 5 . Patients in the MILES group were observed to be able to ambulate a statistically significant longer distance postoperatively immediately, and this advantage lasted from postoperative day 1 until 4 (Fig. 2). They were also able to participate more actively with their physiotherapists and take part in activities involving more rigorous exercises when compared to their counterparts in the control group (Supplementary Fig. $\mathrm{S} 1$ in the online version of this article). On postoperative day $1,54.9 \%$ of patients in the MILES group were able to participate in activities ranging from MOTS to CS, as compared to $42.9 \%$ in the control group $(P=0.007)$. More patients in the control group were only able to contend with more sedentary activities such as RIB to STS in comparison to the MILES group. This advantage of the MILES group was observed from postoperative day 1 to 3 . No differences were observed between the groups for standard outcome measures such as postoperative complications and length of stay. The 30-day readmission rate and need for step-down institutional care remained comparable across the 2 groups.

Table 3 shows the subgroup analysis of patients who underwent surgery for oncological disease. More patients in the MILES group underwent neoadjuvant therapy compared to the control group $(21.7 \%$ vs $12.6 \%$, $P=0.009$ ). There were $50.3 \%$ of patients in the MILES 
Table 1. Baseline demographics and preoperative details of MILES group and control group

\begin{tabular}{|c|c|c|c|}
\hline & $\operatorname{MILES}(\mathbf{N}=\mathbf{3 3 5})$ & Control ( $\mathrm{N}=\mathbf{2 5 6})$ & $P$ value $^{\mathrm{a}}$ \\
\hline Age, mean (SD), years & $72.9(5.6)$ & $73.5(6.1)$ & 0.214 \\
\hline Height, mean (SD), m & $1.59(0.09)$ & $1.59(0.10)$ & 0.934 \\
\hline Preoperative weight, mean (SD), kg & $60.4(12.7)$ & $60.2(12.4)$ & 0.844 \\
\hline Preoperative BMI, mean (SD) & $23.9(4.7)$ & $23.6(4.1)$ & 0.382 \\
\hline $\begin{array}{l}\text { Sex, no. }(\%) \\
\text { Female } \\
\text { Male }\end{array}$ & $\begin{array}{l}136(40.6) \\
199(59.4)\end{array}$ & $\begin{array}{l}110(43.0) \\
146(57.0)\end{array}$ & 0.613 \\
\hline $\begin{array}{l}\text { ASA classification, no. (\%) } \\
1 \\
2 \\
3 \\
4\end{array}$ & $\begin{array}{c}3(0.9) \\
197(58.8) \\
129(38.5) \\
6(1.8)\end{array}$ & $\begin{array}{c}2(0.8) \\
134(52.3) \\
116(45.3) \\
4(1.6)\end{array}$ & 0.428 \\
\hline $\begin{array}{l}\text { Hypertension, no. (\%) } \\
\text { Yes } \\
\text { No }\end{array}$ & $\begin{array}{c}246(73.4) \\
89(26.6)\end{array}$ & $\begin{array}{c}180(71.7) \\
71(28.3)\end{array}$ & 0.708 \\
\hline $\begin{array}{l}\text { Diabetes mellitus, no. (\%) } \\
\text { Yes } \\
\text { No }\end{array}$ & $\begin{array}{l}120(35.8) \\
215(64.2)\end{array}$ & $\begin{array}{c}80(32.1) \\
169(67.9)\end{array}$ & 0.378 \\
\hline $\begin{array}{l}\text { Hyperlipidaemia, no. (\%) } \\
\text { Yes } \\
\text { No }\end{array}$ & $\begin{array}{l}215(64.4) \\
119(35.6)\end{array}$ & $\begin{array}{l}138(55.6) \\
110(44.4)\end{array}$ & 0.039 \\
\hline $\begin{array}{l}\text { Ischaemic heart disease, no. (\%) } \\
\text { Yes } \\
\text { No }\end{array}$ & $\begin{array}{c}77(23.0) \\
258(77.0)\end{array}$ & $\begin{array}{c}51(20.7) \\
195(79.3)\end{array}$ & 0.544 \\
\hline $\begin{array}{l}\text { Stroke, no. (\%) } \\
\text { Yes } \\
\text { No }\end{array}$ & $\begin{array}{c}24(7.2) \\
308(92.8)\end{array}$ & $\begin{array}{c}22(8.9) \\
225(91.1)\end{array}$ & 0.535 \\
\hline $\begin{array}{l}\text { Current smoker, no. (\%) } \\
\text { Yes } \\
\text { No }\end{array}$ & $\begin{array}{c}29(8.7) \\
305(91.3)\end{array}$ & $\begin{array}{c}20(7.9) \\
234(92.1)\end{array}$ & 0.765 \\
\hline $\begin{array}{l}\text { Ex-smoker, no. }(\%) \\
\text { Yes } \\
\text { No }\end{array}$ & $\begin{array}{c}75(22.5) \\
259(77.5)\end{array}$ & $\begin{array}{c}53(20.9) \\
201(79.1)\end{array}$ & 0.687 \\
\hline $\begin{array}{l}\text { ADL-I premorbid, no. (\%) } \\
\text { Yes } \\
\text { No }\end{array}$ & $\begin{array}{c}330(98.8) \\
4(1.2)\end{array}$ & $\begin{array}{c}249(97.3) \\
7(2.7)\end{array}$ & 0.223 \\
\hline $\begin{array}{l}\text { Surgery, no. }(\%) \\
\text { Hepatobiliary and pancreatic } \\
\text { Colorectal }\end{array}$ & $\begin{array}{l}105(31.3) \\
230(68.7)\end{array}$ & $\begin{array}{c}56(21.9) \\
200(78.1)\end{array}$ & 0.012 \\
\hline No. of days from clinic/consent to operation, mean (SD) & $22.5(15.7)$ & $17.3(14.6)$ & 0.001 \\
\hline Preoperative albumin, mean (SD) & $37.2(5.8)$ & $37.6(5.4)$ & 0.539 \\
\hline Preoperative haemoglobin, mean (SD) & $12.0(2.3)$ & $11.9(1.9)$ & 0.575 \\
\hline $\begin{array}{l}\text { Cancer resection, no. (\%) } \\
\text { Yes } \\
\text { No }\end{array}$ & $\begin{array}{c}304(90.7) \\
31(9.3)\end{array}$ & $\begin{array}{c}217(84.8) \\
39(15.2)\end{array}$ & 0.029 \\
\hline Edmonton Frail Scale score, median (IQR) & $3(0-14)$ & & \\
\hline $\begin{array}{l}\text { Physiotherapy referral required, no. (\%) } \\
\text { Yes } \\
\text { No }\end{array}$ & $\begin{array}{l}215(64.2) \\
120(35.8)\end{array}$ & & \\
\hline
\end{tabular}


Table 1. Baseline demographics and preoperative details of MILES group and control group (Cont'd)

\begin{tabular}{|c|c|c|c|}
\hline & MILES (N=335) & Control $(\mathrm{N}=\mathbf{2 5 6})$ & $P$ value ${ }^{\text {a }}$ \\
\hline 3-MinNS score, median (IQR) & $2(0-8)$ & & \\
\hline \multicolumn{4}{|l|}{ Dietician referral required, no. (\%) } \\
\hline Yes & $234(69.9)$ & & \\
\hline No & $101(30.1)$ & & \\
\hline Hand grip strength, mean (SD), kg & $20.3(8.2)$ & & \\
\hline
\end{tabular}

ADL-I: activities of daily living-independent; ASA: American Society of Anesthesiologists; BMI: body mass index; IQR: interquartile range; MILES: Management and Innovation for Longevity in Elderly Surgical patients; 3-MinNS: 3-Minute Nutrition Screening; SD: standard deviation

${ }^{a}$ Bold $P$ values indicate significance

Table 2. Perioperative details of MILES group and control group

\begin{tabular}{|c|c|c|c|}
\hline & MILES (N=335) & Control $(\mathrm{N}=\mathbf{2 5 6})$ & $P$ value ${ }^{a}$ \\
\hline \multicolumn{4}{|c|}{ Final incision is MIS } \\
\hline Yes & $148(44.6)$ & $112(46.1)$ & 0.735 \\
\hline No & $184(55.4)$ & $131(53.9)$ & \\
\hline \multicolumn{4}{|c|}{ Pain score at rest, mean (SD) } \\
\hline POD 1 & $1.7(2.1)$ & $1.4(1.9)$ & 0.089 \\
\hline POD 2 & $0.9(1.6)$ & $0.8(1.5)$ & 0.486 \\
\hline POD 3 & $0.4(1.0)$ & $0.4(1.1)$ & 0.904 \\
\hline POD 4 & $0.3(0.8)$ & $0.3(1.0)$ & 0.643 \\
\hline POD 5 & $0.2(0.7)$ & $0.1(0.6)$ & 0.237 \\
\hline \multicolumn{4}{|c|}{ Pain score on movement, mean (SD) } \\
\hline POD 1 & $4.0(2.4)$ & $3.8(2.2)$ & 0.328 \\
\hline POD 2 & $2.9(2.0)$ & $3.0(2.3)$ & 0.746 \\
\hline POD 3 & $1.8(1.8)$ & $2.0(2.1)$ & 0.266 \\
\hline POD 4 & $1.2(1.6)$ & $1.3(1.7)$ & 0.843 \\
\hline POD 5 & $1.1(1.4)$ & $1.3(1.9)$ & 0.418 \\
\hline \multicolumn{4}{|c|}{ Incentive spirometer volume, $\mathrm{mL}$ (SD) } \\
\hline POD 1 & $677(333)$ & $694(342)$ & 0.647 \\
\hline POD 2 & $683(321)$ & $726(333)$ & 0.256 \\
\hline POD 3 & $731(379)$ & $782(350)$ & 0.316 \\
\hline POD 4 & $707(379)$ & $814(466)$ & 0.134 \\
\hline POD 5 & $752(322)$ & $772(462)$ & 0.765 \\
\hline \multicolumn{4}{|c|}{ Ambulation distance, m (SD) } \\
\hline POD 1 & $30.6(43.0)$ & $16.2(27.8)$ & 0.001 \\
\hline POD 2 & $54.2(58.7)$ & $38.5(49.5)$ & 0.003 \\
\hline POD 3 & $69.6(62.8)$ & $49.4(49.0)$ & 0.001 \\
\hline POD 4 & $65.1(61.9)$ & $48.3(58.4)$ & 0.034 \\
\hline POD 5 & $74.8(74.0)$ & $65.3(70.0)$ & 0.321 \\
\hline \multicolumn{4}{|c|}{ POD 1 physiotherapy, no. (\%) } \\
\hline RIB to STS & $147(45.1)$ & $128(57.1)$ & 0.007 \\
\hline MOTS to CS & $179(54.9)$ & $96(42.9)$ & \\
\hline \multicolumn{4}{|c|}{ POD 2 physiotherapy, no. (\%) } \\
\hline RIB to STS & $60(19.9)$ & 65 (31.9) & 0.002 \\
\hline MOTS to CS & $242(80.1)$ & $139(68.1)$ & \\
\hline \multicolumn{4}{|c|}{ POD 3 physiotherapy, no. (\%) } \\
\hline RIB to STS & $37(13.5)$ & $41(23.7)$ & 0.007 \\
\hline MOTS to CS & $237(86.5)$ & $132(76.3)$ & \\
\hline \multicolumn{4}{|c|}{ POD 4 physiotherapy, no. (\%) } \\
\hline RIB to STS & $34(14.2)$ & $30(21.6)$ & 0.066 \\
\hline MOTS to CS & $206(85.8)$ & $109(78.4)$ & \\
\hline \multicolumn{4}{|c|}{ POD 5 physiotherapy, no. (\%) } \\
\hline RIB to STS & $22(10.6)$ & $23(17.2)$ & 0.101 \\
\hline MOTS to CS & $186(89.4)$ & $111(82.8)$ & \\
\hline
\end{tabular}


Table 2. Perioperative details of MILES group and control group (Cont'd)

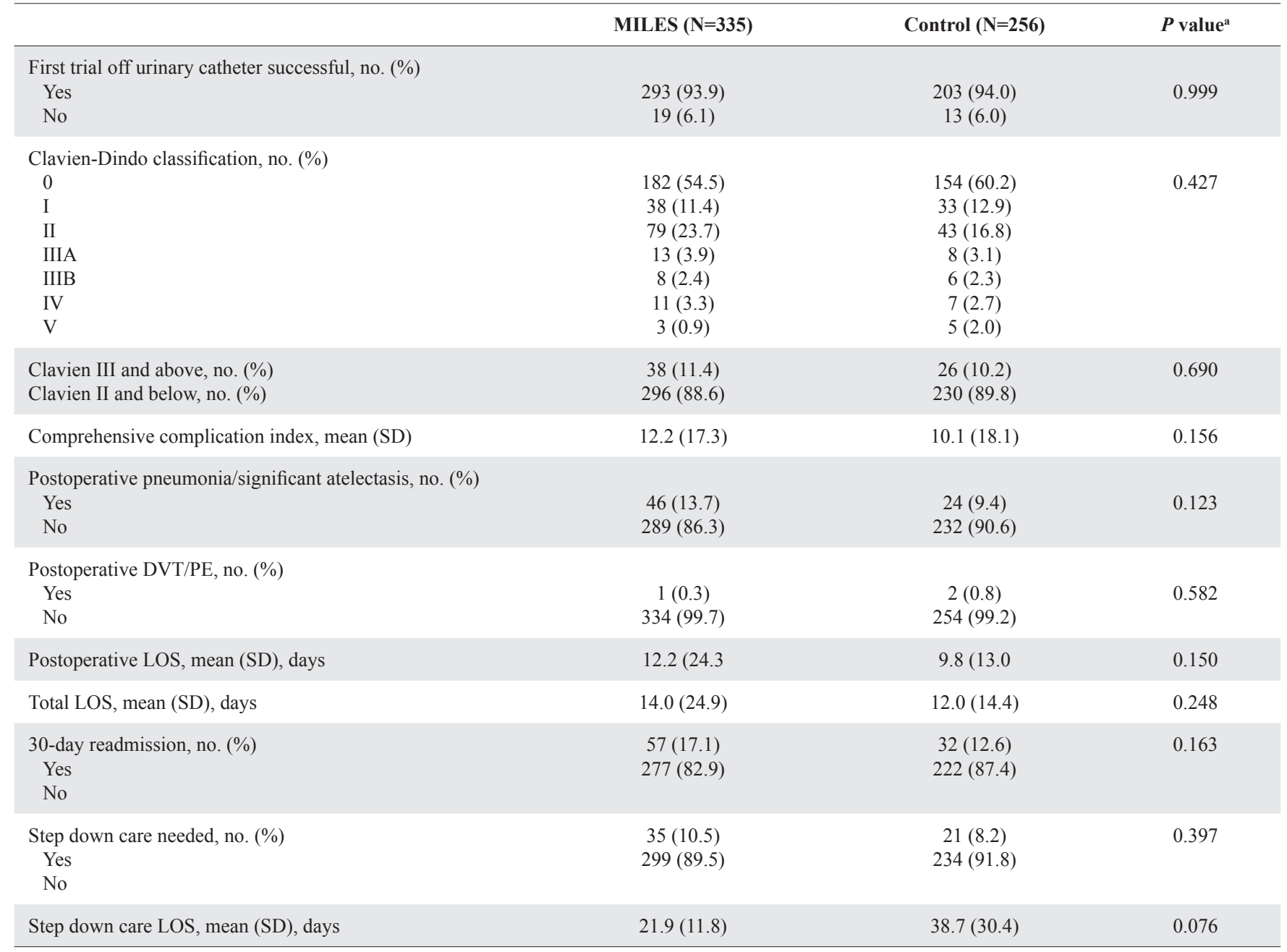

CS: climbing steps; DVT: deep vein thrombosis; LOS: length of stay; MILES: Management and Innovation for Longevity in Elderly Surgical patients; MIS: minimally invasive surgery; MOTS: moving on the spot; PE: pulmonary embolism; POD: postoperative day; RIB: resting in bed; SD: standard deviation; STS: sit to stand

${ }^{a}$ Bold $P$ values indicate significance

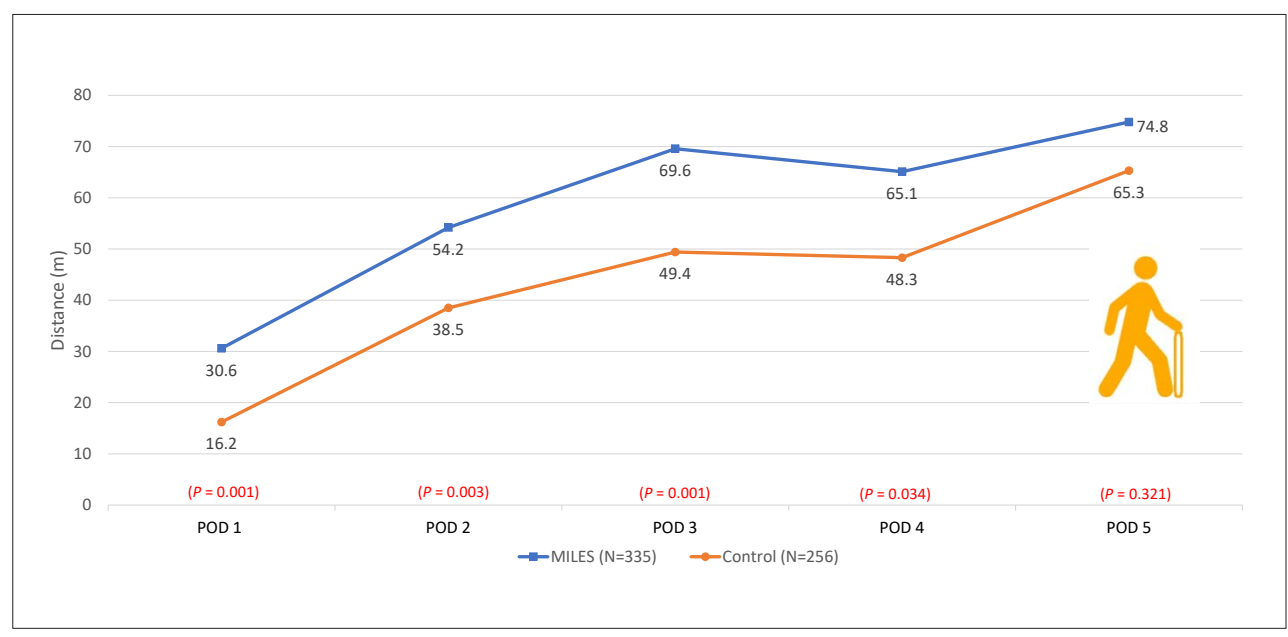

Fig. 2. Postoperative ambulation distance in metres.

MILES: Management and Innovation for Longevity in Elderly Surgical patients; POD: postoperative day 
Table 3. Subgroup analysis of patients who underwent cancer resection surgery

\begin{tabular}{|c|c|c|c|}
\hline & MILES (n=304) & Control $(n=217)$ & $P$ value $^{\mathrm{a}}$ \\
\hline \multicolumn{4}{|l|}{ Surgery, no. (\%) } \\
\hline Hepatobiliary and pancreatic & $95(31.3)$ & $48(22.1)$ & 0.022 \\
\hline Colorectal & $209(68.8)$ & $169(77.9)$ & \\
\hline \multicolumn{4}{|l|}{ Metastatic disease (preoperation), no. (\%) } \\
\hline Yes & $28(9.2)$ & $19(9.0)$ & 0.999 \\
\hline No & $276(90.8)$ & $192(91.0)$ & \\
\hline \multicolumn{4}{|l|}{ Neoadjuvant chemotherapy, no. (\%) } \\
\hline Yes & 64 (21.7) & $26(12.6)$ & 0.009 \\
\hline No & $231(78.3)$ & $181(87.4)$ & \\
\hline Surgery to adjuvant chemotherapy, mean (SD), days & $61.7(26.4)$ & $51.2(17.7)$ & 0.001 \\
\hline \multicolumn{4}{|l|}{ Adjuvant chemotherapy required, no. (\%) } \\
\hline Yes & $152(50.3)$ & $95(44.2)$ & 0.181 \\
\hline No & $150(49.7)$ & $120(55.8)$ & \\
\hline \multicolumn{4}{|l|}{ Initiated adjuvant chemotherapy, no. (\%) } \\
\hline Yes & $129(87.2)$ & $61(65.6)$ & $<0.001$ \\
\hline No & $19(12.8)$ & $32(34.4)$ & \\
\hline \multicolumn{4}{|l|}{ Adherence to chemotherapy initiation date, no. (\%) } \\
\hline Yes & $121(93.8)$ & $58(95.1)$ & 0.999 \\
\hline No & $8(6.2)$ & $3(4.9)$ & \\
\hline \multicolumn{4}{|l|}{ Completed chemotherapy, no. (\%) } \\
\hline Yes & $71(77.2)$ & $49(81.7)$ & 0.548 \\
\hline No & $21(22.8)$ & $11(18.3)$ & \\
\hline
\end{tabular}

MILES: Management and Innovation for Longevity in Elderly Surgical patients; SD: standard deviation

a Bold $P$ values indicate significance

group who required adjuvant therapy (based on final histology and MTB discussion), being comparable to $44.2 \%$ in the control group $(P=0.181)$. Patients who have undergone the MILES programme were statistically more likely to go on to successful adjuvant chemotherapy initiation (MILES $87.2 \%$ vs control $65.6 \%, P<0.001$ ). Among patients who initiated chemotherapy, the adherence to the planned chemotherapy initiation date and the proportion of patients who completed chemotherapy were comparable between the 2 groups ( $P=0.999$ and $P=0.548$, respectively). Further analysis with a multivariate model (Table 4) revealed neoadjuvant treatment to be significantly associated with the odds of the patient being recommended for adjuvant treatment.

Organ-specific subgroup analysis was performed (Supplementary Tables S1, S2 and S3 in the online version of this article). Results showed similar trends as described above, although the reduced sample size within each subgroup limited interpretation due to a diminished statistical power. Further analysis was performed by including only patients who had at least 2 weeks from date of clinic/consent to operation (Supplementary Table S4 in the online version of this article). Among patients who required step down care, MILES patients required a shorter length of stay compared to control, 21.6 days, SD 12.0 vs 37.5 days, SD 18.4 $(P=0.015)$.

\section{DISCUSSION}

In line with an ageing population, the number of older patients requiring major surgeries has been increasing rapidly. Research into perioperative strategies specific to the older population has become of paramount importance. Prehabilitation is emerging as a potential strategy that may help improve the surgery experience in older patients. The current study reaffirmed the notion that prehabilitation can positively contribute to the recovery of an older patient in the immediate postoperative period. Although most of the surgical outcome measures such as length of stay and postoperative complications were comparable across both groups, patients who have undergone prehabilitation were able to mobilise better, faster and further compared to the control group. This was hardly surprising as numerous studies have reported improved 6-minute walk tests (6MWTs) and cardiorespiratory fitness in patients who have undergone prehabilitation., ${ }^{5,6,21-23}$ 
Table 4. Univariate and multivariate analyses of variables potentially associated with initiation of adjuvant chemotherapy (in the cancer resection patient subgroup)

\begin{tabular}{|c|c|c|c|c|c|c|}
\hline \multirow[t]{2}{*}{ Variables } & \multicolumn{3}{|c|}{ Univariate } & \multicolumn{3}{|c|}{ Multivariate } \\
\hline & OR & $95 \%$ CI & $P$ value ${ }^{a}$ & OR & $95 \%$ CI & $P$ value $^{\mathrm{a}}$ \\
\hline MILES Group & 4.84 & $2.11-11.09$ & $<0.001$ & 3.22 & $1.66-6.27$ & 0.001 \\
\hline Hepatobiliary and pancreatic patients & 2.83 & $1.13-7.12$ & 0.027 & & & \\
\hline Neoadjuvant treatment & 9.31 & $2.73-31.77$ & $<0.001$ & 5.00 & $1.69-14.75$ & 0.004 \\
\hline Days from clinic/consent to operation & 0.974 & $0.95-1.00$ & 0.082 & & & \\
\hline MIS incision & 1.72 & $0.77-3.84$ & 0.188 & & & \\
\hline POD 1 ambulation distance & 0.99 & $0.97-1.01$ & 0.268 & & & \\
\hline POD 1 MOTS to CS & 2.88 & $0.85-9.76$ & 0.089 & & & \\
\hline Clavien III and above complications & 0.30 & $0.08-1.10$ & 0.070 & & & \\
\hline Step down care needed & 1.70 & $0.49-5.92$ & 0.405 & & & \\
\hline Metastatic disease (preoperation) & 0.31 & $0.10-1.01$ & 0.051 & & & \\
\hline Hyperlipidaemia & 0.93 & $0.42-2.09$ & 0.861 & & & \\
\hline
\end{tabular}

CI: confidence interval; CS: climbing steps; MILES: Management and Innovation for Longevity in Elderly Surgical patients; MIS: minimally invasive surgery; MOTS: moving on the spot; OR: odds ratio; POD: postoperative day

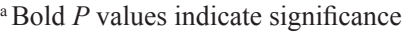

Our findings were concordant with the published literature as it was reasonable to assume that postoperative ambulation distance correlated with the 6MWT.

The theory that improved mobilisation leads to decreased postoperative complications related to immobility is one of the cornerstones of prehabilitation interest. Nevertheless, there exists conflicting evidence for prehabilitation leading to improved postoperative recovery in terms of traditional outcome measures such as postoperative complications and length of stay. Some studies suggested a modest impact on postoperative morbidity ${ }^{8,24-26}$ while other reviews concluded that more evidence was needed. ${ }^{9-10,27-29}$ This would appear counterintuitive when prehabilitation can have benefits on the physical preparedness of the patient scheduled for a major operation. In our opinion, the lack of convincing data in the literature to date may be related to the selection of patients for prehabilitation and the selection of outcome measures. In our retrospective study, as frailty assessment was not performed for the control group, we were unable to perform a subgroup analysis comparing only frail patients between the 2 groups. This subgroup comparison would have provided fresh insights as the frailest patients would conceivably benefit most from prehabilitation. An analysis of the frailest patients in a randomised controlled trial may reveal statistically significant advantages of prehabilitation through traditional surgical outcome measures such as length of stay and postoperative major complications. However, there are logistical and ethical challenges to overcome in designing such a trial that are beyond the scope of this discussion. The clinical significance of improved postoperative mobility in older patients with prehabilitation in our study remains to be seen and would require carefully designed large prospective trials to validate.

Patients who underwent MILES prehabilitation were more likely to adhere to the recommended postoperative adjuvant treatment even after accounting for potential confounders in a multivariate analysis. To the best of our knowledge, this is the first study to report a greater adherence to adjuvant treatment in a prehabilitation cohort. Interestingly, Trepanier et al. highlighted an improved 5-year disease-free survival in stage III colorectal cancer patients who had undergone prehabilitation compared to those who had not. ${ }^{30}$ As our study was a retrospective study with inherent limitations, our findings were clearly insufficient to demonstrate causality. Potential non-quantifiable confounders, such as extent of social support and functional status, were not adjusted for with the multivariate model. Further prospective studies investigating this association will be required to establish causality. 
The motivation to adhere to adjuvant therapy may very well be psychosocial in nature. For example, patients who have undergone prehabilitation may feel psychologically better prepared for treatment in the long haul, with an understanding that cancer treatment is a marathon rather than a sprint. This would have translated to increased mental fortitude to soldier on with a recommended adjuvant treatment. Future qualitative studies to investigate psychosocial factors would prove useful. Early enrolment into a prehabilitation programme may aid to expose older patients to the healthcare workers in charge of their care early in the medical journey. This would allow for good rapport to be established and the fostering of a greater sense of trust in the healthcare team, thereby influencing a patient's decision to accept a recommended cancer treatment. We were unable to perform a survival analysis as patients whose surgeries were performed until the reporting of the current study were included.

In addition to prehabilitation, our multivariate model suggested that neoadjuvant therapy was strongly associated with the odds of being compliant to postoperative adjuvant treatment. Factors affecting compliance to adjuvant therapy have been studied; however, most literature on the topic recommended further larger scale, prospective research. ${ }^{31,32}$ This was especially so for the subgroup of older, postoperative patients. A systematic review by Puts et al. found that factors for accepting cancer treatment included convenience and success rates of treatment, appreciating the necessity of treatment, and sufficient trust in the physician to follow a recommended course of treatment. ${ }^{33}$ Factors that may result in declining cancer treatment included concerns about the discomfort of the treatments, fear of side effects and transportation difficulties. This review was conducted on all cancer patients who were older, but not specifically in the postoperative setting. The findings of Puts et al. suggested that patients who have undergone neoadjuvant treatment were less fearful of the discomfort of the treatment and more convinced about the efficacy of adjuvant treatment, leading to increased compliance. This could explain why neoadjuvant therapy was found to be an independent predictor of compliance to adjuvant treatment in our multivariate analysis, in addition to prehabilitation. A positive synergy between neoadjuvant therapy and prehabilitation may be established. Neoadjuvant therapy provided the cancer patient with more time for prehabilitation, and together, these 2 factors led to increased compliance to adjuvant treatment. Frail patients who would likely benefit most from prehabilitation should be considered for neoadjuvant therapy if appropriate for the underlying disease, even if routine care suggested for upfront resection (e.g. upfront resectable pancreatic cancer).

\section{Limitations}

Inherent to its retrospective nature, the current study had several limitations. It was difficult to ascertain the compliance rate of our prehabilitation programme, as most of the physical exercises and nutritional advice were carried out at home after a session with the respective specialists. This would potentially lead to heterogeneous exposure to actual prehabilitation in our MILES cohort. A specially trained nurse served as the unit coordinator and had followed up actively with prehabilitation patients to increase compliance to prehabilitation measures. Compliance to physical prehabilitation was an issue that many prehabilitation programmes across the world face $^{34}$ and may be heavily dependent on whether the prehabilitation was carried out in the hospital or at home. Its significance, however, may be somewhat diminished in an intention-to-treat analysis.

The length of time between the clinic consultation, where the decision for surgery was made, and the surgery date was not fixed. Therefore, some patients in the MILES group may have had limited exposure to prehabilitation. Despite this and the lack of data on compliance, both of which would have resulted in an underestimation of the prehabilitation effect, we observed a significant improvement in postoperative compliance to adjuvant therapy and an ability to mobilise in the MILES group. Additionally, subgroup analysis of patients who were 2 weeks or more from clinic consultation to surgery demonstrated a shorter duration of step-down care in the MILES group when compared to control. This suggested that if a significant length of time was spent in prehabilitation, the duration of rehabilitation required postoperation may be shortened. Future large-scale studies would be required to investigate this association.

\section{CONCLUSION}

There were no differences in traditional surgical outcome measures such as length of stay or postoperative complications between older patients who have undergone prehabilitation and control group. There was an increase in patient mobility in the immediate postoperative period with prehabilitation, as well as an association between prehabilitation and increased adherence to postoperative adjuvant therapy. Larger prospective studies with a focus on specific subgroups such as the frailest patients will be needed to validate the findings of this retrospective review. 


\section{REFERENCES}

1. Al-Refaie WB, Parsons HM, Henderson WG, et al. Major cancer surgery in the elderly: results from the American College of Surgeons National Surgical Quality Improvement Program. Ann Surg 2010;251:311-8.

2. Morley JE, Vellas B, van Kan GA, et al. Frailty consensus: a call to action. J Am Med Dir Assoc 2013;14:392-7.

3. Lawrence VA, Hazuda HP, Cornell JE, et al. Functional independence after major abdominal surgery in the elderly. J Am Coll Surg 2004; 199:762-72.

4. Carli F, Charlebois P, Stein B, et al. Randomized clinical trial of prehabilitation in colorectal surgery. Br J Surg 2010;97:1187-97.

5. Gillis $\mathrm{C}, \mathrm{Li} \mathrm{C}$, Lee $\mathrm{L}$, et al. Prehabilitation versus rehabilitation: a randomized control trial in patients undergoing colorectal resection for cancer. Anesthesiology 2014;121:937-47.

6. Bousquet-Dion G, Awasthi R, Loiselle SE, et al. Evaluation of supervised multimodal prehabilitation programme in cancer patients undergoing colorectal resection: a randomized control trial. Acta Oncol 2018;57:849-59.

7. Li C, Carli F, Lee L, et al. Impact of a trimodal prehabilitation program on functional recovery after colorectal cancer surgery: a pilot study. Surg Endosc 2013;27:1072-82.

8. Moran J, Guinan E, McCormick P, et al. The ability of prehabilitation to influence postoperative outcome after intraabdominal operation: a systematic review and meta-analysis. Surgery 2016;160:1189-201

9. Hijazi Y, Gondal U, Aziz O. A systematic review of prehabilitation programs in abdominal cancer surgery. Int J Surg 2017;39:156-62.

10. Bruns ER, van den Heuvel B, Buskens CJ, et al. The effects of physical prehabilitation in elderly patients undergoing colorectal surgery: a systematic review. Colorectal Dis 2016;18:0267-77.

11. Levett DZH, Grimmett C. Psychological factors, prehabilitation and surgical outcomes: evidence and future directions. Anaesthesia 2019;74 Suppl 1:36-42.

12. Scheede-Bergdahl C, Minnella EM, Carli F. Multi-modal prehabilitation: addressing the why, when, what, how, who and where next? Anaesthesia 2019;74 Suppl 1:20-6.

13. McIsaac DI, Jen T, Mookerji N, et al. Interventions to improve the outcomes of frail people having surgery: a systematic review. PLoS One 2017;12:e0190071.

14. Carli F, Bousquet-Dion G, Awasthi R, et al. Effect of Multimodal Prehabilitation vs Postoperative Rehabilitation on 30-Day Postoperative Complications for Frail Patients Undergoing Resection of Colorectal Cancer-A Randomized Clinical Trial. JAMA Surg 2020;155:233-42.

15. Slankamenac K, Graf R, Barkun J, et al. The comprehensive complication index: a novel continuous scale to measure surgical morbidity. Ann Surg 2013;258:1-7.

16. Rolfson DB, Majumdar SR, Taher A, et al. Development and validation of a new instrument for frailty. Clin Invest Med 2000;23:336.

17. Rolfson DB, Majumdar SR, Tsuyuki RT, et al. Validity and reliability of the Edmonton Frail Scale. Age Ageing 2006;35:526-9.
18. Lim SL, Tong CY, Ang E, et al. Development and validation of 3-Minute Nutrition Screening (3-MinNS) tool for acute hospital patients in Singapore. Asia Pac J Clin Nutr 2009;18:395-403.

19. Makhija S, Baker J. The Subjective Global Assessment: a review of its use in clinical practice. Nutr Clin Pract 2008;23:405-9.

20. Lim SL, Lin XH, Daniels L. Seven-Point Subjective Global Assessment Is More Time Sensitive Than Conventional Subjective Global Assessment in Detecting Nutrition Changes. JPEN J Parenter Enteral Nutr 2016 Sep;40:966-72.

21. Minnella EM, Awasthi R, Bousquet-Dion G, et al. Multimodal prehabilitation to enhance functional capacity following radical cystectomy: a randomized controlled trial. Eur Urol Focus 2021;7:132-8.

22. Minnella EM, Awasthi R, Loiselle SE, et al. Effect of exercise and nutrition prehabilitation on functional capacity in esophagogastric cancer surgery: a randomized clinical trial. JAMA Surg 2018; 153:1081-9.

23. Barberan-Garcia A, Ubré M, Roca J, et al. Personalised prehabilitation in high-risk patients undergoing elective major abdominal surgery: a randomized blinded controlled trial. Ann Surg 2018;267:50-6.

24. Gillis C, Buhler K, Bresee L, et al. Effects of nutritional prehabilitation, with and without exercise, on outcomes of patients who undergo colorectal surgery: a systematic review and metaanalysis. Gastroenterology 2018;155:391-410.e4.

25. Heger P, Probst P, Wiskemann J, et al. A systematic review and metaanalysis of physical exercise prehabilitation in major abdominal surgery (PROSPERO 2017 CRD42017080366). J Gastrointest Surg 2020;24:1375-85

26. Hughes MJ, Hackney RJ, Lamb PJ, et al. Prehabilitation Before Major Abdominal Surgery: A Systematic Review and Meta-analysis. World J Surg 2019;43:1661-68.

27. Luther A, Gabriel J, Watson RP, et al. The Impact of Total Body Prehabilitation on Post-Operative Outcomes After Major Abdominal Surgery: A Systematic Review. World J Surg 2018;42:2781-91.

28. Bolshinsky V, Li MH, Ismail $\mathrm{H}$, et al. Multimodal prehabilitation programs as a bundle of care in gastrointestinal cancer surgery: a systematic review. Dis Colon Rectum 2018;61:124-38.

29. Milder DA, Pillinger NL, Kam PCA. The role of prehabilitation in frail surgical patients: A systematic review. Acta Anaesthesiol Scand 2018;62:1356-66

30. Trépanier M, Minnella EM, Paradis T, et al. Improved disease-free survival after prehabilitation for colorectal cancer surgery. Ann Surg 2019;270:493-501.

31. Jorgensen ML, Young JM, Solomon MJ. Adjuvant chemotherapy for colorectal cancer: age differences in factors influencing patients' treatment decisions. Patient Prefer Adherence 2013;7:827-34.

32. Puts MTE, Monette J, Girre V, et al. Characteristics of older newly diagnosed cancer patients refusing cancer treatments. Support Care Cancer 2010;18:969-74.

33. Puts MT, Tapscott B, Fitch M, et al. A systematic review of factors influencing older adults' decision to accept or decline cancer treatment. Cancer Treat Rev 2015;41:197-215.

34. Ferreira V, Agnihotram RV, Bergdahl A, et al. Maximizing patient adherence to prehabilitation: What do the patients say? Support Care Cancer 2018;26:2717-23. 